\title{
IMPLEMENTATION OF SHIP INTRUSION DETECTION AND MONITORING SYSTEM BY LabVIEW
}

\author{
R.M.Madhumathi ${ }^{1}$, Dr.A.Jagadeesan ${ }^{2}$ \\ First year M.E (Instrumentation Engineering), Bannari Amman Institute of Technology, Sathyamangalam, India ${ }^{1}$ \\ Asst. Prof. (Senior Grade), Department of EIE, Bannari Amman Institute of Technology, Sathyamangalam, India ${ }^{2}$
}

\begin{abstract}
Surveillance is a critical problem for harbor protection, border control or the security of commercial facilities. The effective protection of vast near-coast sea surfaces and busy harbor areas from intrusions of unauthorized marine vessels, such as pirates, smugglers, illegal fishermen is particularly challenging. The main aim is to detect the ship, which cross over the border or secured industries using ultrasonic sensor and axis sensor. These sensors are placed on grid for every $40 \mathrm{~km}$ apart when the intrusion is passed away the sensor, it senses the obstacle and measures the intruder angle and distance. This will be displayed in the form of graphical representation in LabVIEW (Laboratory Virtual Instrumentation Engineers Workbench). An alert message will be sending to control room using GSM (Global System for Mobile communication).
\end{abstract}

Keywords-Wireless Sensor Networks (WSN), Synthetic Aperture Radar, LabVIEW, PIC Microcontroller, Global System for Mobile communication (GSM)

\section{INTRODUCTION}

This paper presents an innovative solution for ship such as those involved in piracy activity. There are two intrusion detection. Equipped with ultrasonic sensors, fundamentally different means of detecting ships in SAR deploy an experimental WSN (Wireless Sensor Networks) imagery, detection of the ship target itself and detection of on the sea's surface to detect ships. This will be intimated the ship wake. They note that most current research on to the microcontroller by emitting digital signal from the ship detection is based on detecting the ship rather than its sensors. . These digital signals will be formed as a packet wake. First of all, the reflected signal from the ship as a consisting of date, time and node id will be sent to the feature is more useful and less dependent on the sea state server node using Zigbee. The server will receive this and higher than the surrounding sea clutter. Moreover, the signal using Zigbee in the receiving side and it will be ship wakes are often invisible for some special angles of displayed in LabVIEW software. An alert message will be radar and the detection of ship wakes needs heavy sent to control room using GSM. The conducted computational processing. Consequently, in this paper, evaluations with real data collected in our initial they focus on the schemes of detecting ship targets experiments, and provide quantitative analysis of the themselves rather than ship wakes since ships have more detection system, such as the successful detection ratio, robust back-scattering properties than ship wakes, and ship detection latency, and an estimation of an intruding wakes do not exist sometimes. Due to the corner reflection vessel's velocity.

\section{LITERATURE REVIEW}

One of the most common active sensors for imaging operation is SAR (Synthetic Aperture Radar). In recent years, high-resolution SAR images are widely used both in military and civilian applications. Much effort has been devoted to ship detection using SAR images. Because SAR images are less influenced by the time and weather conditions than the optical images, they are more suitable for ship detection. Systems using this technique can efficiently perform the monitoring and surveillance mission of the sea surface. Ship detection is of great usefulness former time surveillance, fishery activity management, monitoring ship traffic, and in particular, increasing security by monitoring illegally operating ships,

from the ship structure or between the ship body and ocean surface, ship targets are usually clear in SAR images. When the ocean surface is relatively calm (e.g., when the wind speed is lower than two meters per second), the reflection of the ocean surface to the radar wave is specular reflection and the echo signal is very faint. In most research, the background of the SAR images is quite dark and targets are bright, so it is very easy to detect these ship targets. The backscattering echo will be very strong and the whole SAR image will be rather bright. This causes many difficulties in the detection of the ships, and especially small ships. The graphical approach also allows non-programmers to build programs by dragging and dropping virtual representations of lab equipment with which they are already familiar. The LabVIEW programming environment, with the included examples and documentation, makes it simple to create small applications. 


\section{EXISTING METHOD}

RADAR (RAdio Detection And Ranging) method is used to detect the intrusion of ship. The back ground of SAR image is quite dark and the targets are bright so it is easy to detect the object, when the wind is fierce, large waves will be stirred. The backscattering echo will be very strong. This causes many difficulties in the detection of the ships, and especially small ships. The overall accuracy is poor and it is difficult to detect the ship during bad weather condition.

\section{PROPOSED METHOD}

In this paper, WSN is used to detect the intrusion on the basis of distance and angle. Here one input is given as ultrasonic sensor which is used to detect the obstacle in the sea and measures the distance. Another input accelerometer sensor is sensing transducer that provides an output proportional to acceleration vibration and shock.

\subsection{Working Model of Proposed Method}

It is used to detect the obstacle in the sea and measure the distance. Here by using step down transformer to convert $230 \mathrm{~V}$ AC supply into $12 \mathrm{~V}$ AC supply. Using the bridge rectifier, AC signal is converted to DC signal and to reduce the ripple, capacitor of $1000 \mu \mathrm{f}$ is used. IC 7805 voltage regulator is used for converting the $12 \mathrm{~V}$ into $5 \mathrm{~V}$ for the input. The input of the ultrasonic sensor and accelerometer sensor is given to the microcontroller. If the intruder is present, the distance of the obstacle and the measured angle is displayed in LCD. Power driver is used to convert low voltage into high voltage for indication. From the microcontroller the output signal is transmitted through Zigbee and this signal will be intimated to the microcontroller by emitting digital signal from the sensors. These digital signals will be formed as a packet consisting of date, time and node id will be sent to the server node using Zigbee. The server will receive this signal from Zigbee in the receiver side and it will be displayed by LabVIEW software. An alert message will be sent to control room using GSM and the database can be maintained in the personal computer.

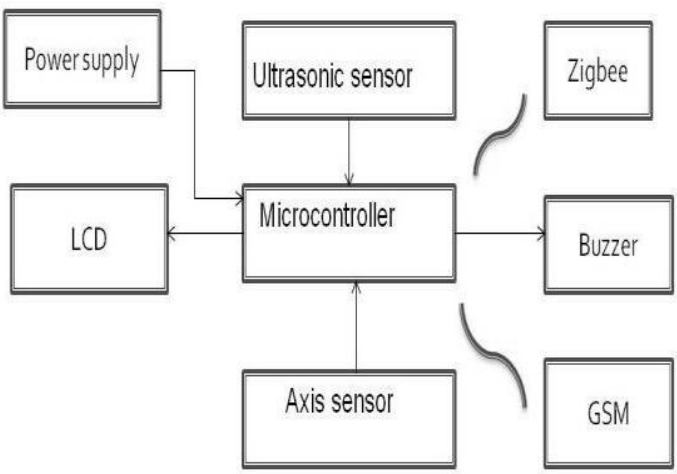

Fig. 1 Block Diagram of Transmitter Node
In the Transmitter node the real time signals such as movements, sounds, and temperature are given to the microcontroller, it converts them as digital signal. The corresponding voltage signals are transmitted through ZigBee. An alert message is sent to mobile using GSM.

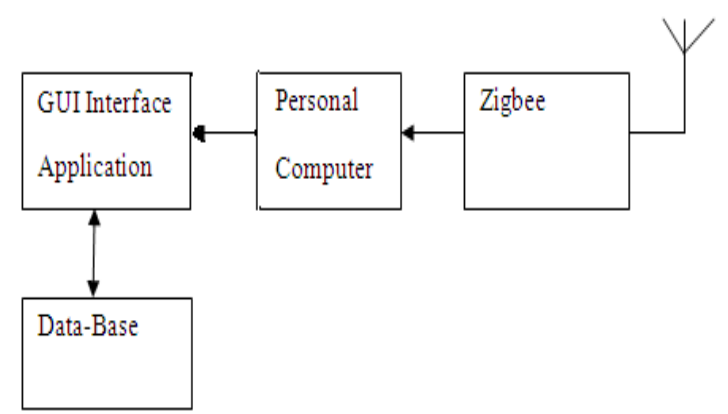

Fig. 2 Block Diagram of Receiver Node

The receiver node of the zigbee receives the signal from zigbee transmitter and displayed in the LabVIEW. The signals from the zigbee are passed to SIM (Subscriber Identity Module) card so that an alert message is given to the coastal area control room officers. The data base is maintained in the computer for the references.

\subsubsection{Ultrasonic Sensor}

Ultrasonic sensors (also known as transceivers when they both send and receive) work on a principle similar to radar or sonar which evaluate attributes of a target by interpreting the echoes from radio or sound waves respectively. Ultrasonic sensors generate high frequency sound waves and evaluate the echo which is received back by the sensor. Sensors calculate the time interval between sending the signal and receiving the echo to determine the distance to an object. This technology can be used for measuring wind speed and direction (anemometer), fullness of a tank and speed through air or water. For measuring speed or direction a device uses multiple detectors and calculates the speed from the relative distances to particulates in the air or water. To measure the amount of liquid in a tank, the sensor measures the distance to the surface of the fluid. Further applications include: humidifiers, sonar, medical ultrasonography, burglar alarms and non-destructive testing.

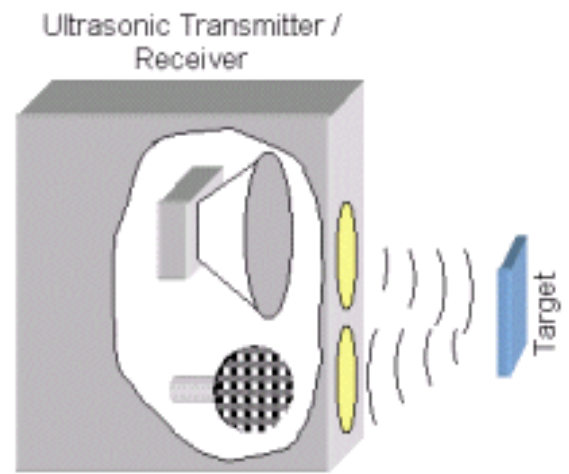

Fig.3 Ultrasonic Transmitter /Receiver 
Systems typically use a transducer which generates sound waves in the ultrasonic range, above $18,000 \mathrm{~Hz}$, by turning electrical energy into sound, then upon receiving the echo turn the sound waves into electrical energy which can be measured and displayed. The technology is limited by the shapes of surfaces and the density or consistency of the material. For example foam on the surface of a fluid in a tank could distort a reading. An ultrasonic transducer is a device that converts energy into ultrasound, or sound waves above the normal range of human hearing. While technically a dog whistle is an ultrasonic transducer that converts mechanical energy in the form of air pressure into ultrasonic sound waves, the term is more to be used to refer to piezoelectric transducers that convert electrical energy into sound. Piezoelectric crystals have the property of changing size when a voltage is applied, thus applying an $\mathrm{AC}$ across them causes them to oscillate at very high frequencies, thus producing very high frequency sound waves. The location at which a transducer focuses the sound can be determined by the active transducer area and shape, the ultrasound frequency, and the sound velocity of the propagation medium.

\subsubsection{Accelerometer Sensor}

In this accelerometer sensor is also known as axis sensor. Whenever a structure moves, it experiences acceleration. Measuring this acceleration promotes an understanding of the dynamic characteristics that govern the object's behavior. The data can be used to modify response, enhance ruggedness, improve durability, or reduce noise and vibration. Accelerometers are sensing transducers that provide an output proportional to acceleration, vibration, and shock. The piezoelectric accelerometer is the most popular class of these devices.

$$
\omega=\sqrt{\frac{k}{m}}
$$

The other types are based on piezoresistive, capacitive, and servo technologies. An accelerometer is a device that measures proper acceleration. The proper acceleration measured by an accelerometer is not necessarily the coordinate acceleration (rate of change of velocity). Instead, the accelerometer sees the acceleration associated with the phenomenon of weight experienced by any test mass at rest in the frame of reference of the accelerometer device. Highly sensitive accelerometers are components of inertial navigation systems for aircraft and missiles. Accelerometers are used to detect and monitor vibration in rotating machinery. Accelerometers are used in tablet computers and digital cameras so that images on screens are always displayed upright. The ADXL335 complete, low-power 3-axis accelerometer measures dynamic acceleration (motion, shock, or vibration) and static acceleration (tilt or gravity) over a $\pm 3 g$ range with $0.3 \%$ nonlinearity and $0.01 \% /{ }^{\circ} \mathrm{C}$ temperature stability.

\subsubsection{Zigbee}

ZigBee is a specification for a suite of high level communication protocols using small, low-power digital radios based on an IEEE 802 standard for personal area networks. Applications include wireless light switches, electrical meters with in-home-displays, and other consumer and industrial equipment that require shortrange wireless transfer of data at relatively low rates. ZigBee is targeted at RF applications and secure networking. ZigBee has a defined rate of $250 \mathrm{kbps}$ best suited for periodic or intermittent data or a single signal transmission from a sensor or input device. ZigBee is a low-cost, low-power, wireless mesh network standard. The low cost allows the technology to be widely deployed in wireless control and monitoring applications. Low powerusage allows longer life with smaller batteries. Mesh networking provides high reliability and more extensive range. ZigBee operates in the ISM radio bands $868 \mathrm{MHz}$ in Europe, $915 \mathrm{MHz}$ in the USA and Australia, and 2.4 $\mathrm{GHz}$ in most jurisdictions worldwide. Data transmission rates vary from 20 to 900 kilobits/second. The ZigBee network layer natively supports both star and tree typical networks, and generic mesh networks. Every network must have one coordinator device, tasked with its creation, the control of its parameters and basic maintenance. Within star networks, the coordinator must be the central node. Both trees and meshes allow the use of ZigBee routers to extend communication at the network level. ZigBee protocol stack ZigBee builds upon the physical layer and medium access control defined in IEEE standard 802.15.4 (2003 version) for low-rate WPANs. The specification goes on to complete the standard by adding four main components network layer, application layer, ZDOs and manufacturer-defined application objects which allow for customization and favor total integration. Besides adding two high-level network layers to the underlying structure, the most significant improvement is the introduction of ZDOs. These are responsible for a number of tasks, which include keeping of device roles, management of requests to join a network, device discovery and security. ZigBee nodes can go from sleep to active mode in $30 \mathrm{~ms}$ or less, the latency can be low and devices can be responsive, particularly compared to Bluetooth wake-up delays, which are typically around three seconds. ZigBee nodes can sleep most of the time average power consumption can be low, resulting in long battery life.

\subsubsection{GSM Technology}

GSM is a cellular network, which means that cell phones connect to it by searching for cells in the immediate vicinity. There are five different cell sizes in a GSM network macro, micro, Pico, femto and umbrella cells. The coverage area of each cell varies according to the implementation environment. Macro cells can be regarded as cells where the station antenna is installed on a mast or a building above average rooftop level. Micro cells are cells whose antenna height is under average rooftop level, they are typically used in urban areas. Pico cells are small cells whose coverage diameter is a few dozen meters they are mainly used indoors. Femto cells 
are cells designed for use in residential or small business environments and connect to the service provider's network via a broadband internet connection. Umbrella cells are used to cover shadowed regions of smaller cells and fill in gaps in coverage between those cells.

Cell horizontal radius varies depending on antenna height, antenna gain, and propagation conditions from a couple of hundred meters to several tens of kilometers. The longest distance the GSM specification supports in practical use is 35 kilometers.

\section{Circuit Diagram of Proposed Method}

It describes the overall circuit diagram of the Transmitter node. The inputs to the micro controller are analog signals fed by the two sensors namely ultrasonic and accelerometer sensor. In this, sound wave is detected by ultrasonic sensor when the ship arrives. The output of ultrasonic sensors are digital. The range of the sensor used here is 1 feet.The output signals of the sensors are connected to RB0 and RB1 of micro controller. The user selects the bandwidth of the accelerometer using the $\mathrm{C}_{\mathrm{X}}$, $\mathrm{C}_{\mathrm{Y}}$, and $\mathrm{C}_{\mathrm{Z}}$ capacitors at the $\mathrm{X}_{\mathrm{OUT}}, \mathrm{Y}_{\mathrm{OUT}}$, and $\mathrm{Z}_{\mathrm{OUT}}$ pins. Measurement bandwidth can be selected to suit the application from $0.5 \mathrm{~Hz}$ to $1600 \mathrm{~Hz}$ for $\mathrm{X}$ - and $\mathrm{Y}$ - axes and from $0.5 \mathrm{~Hz}$ to $550 \mathrm{~Hz}$ for the Z-axis.

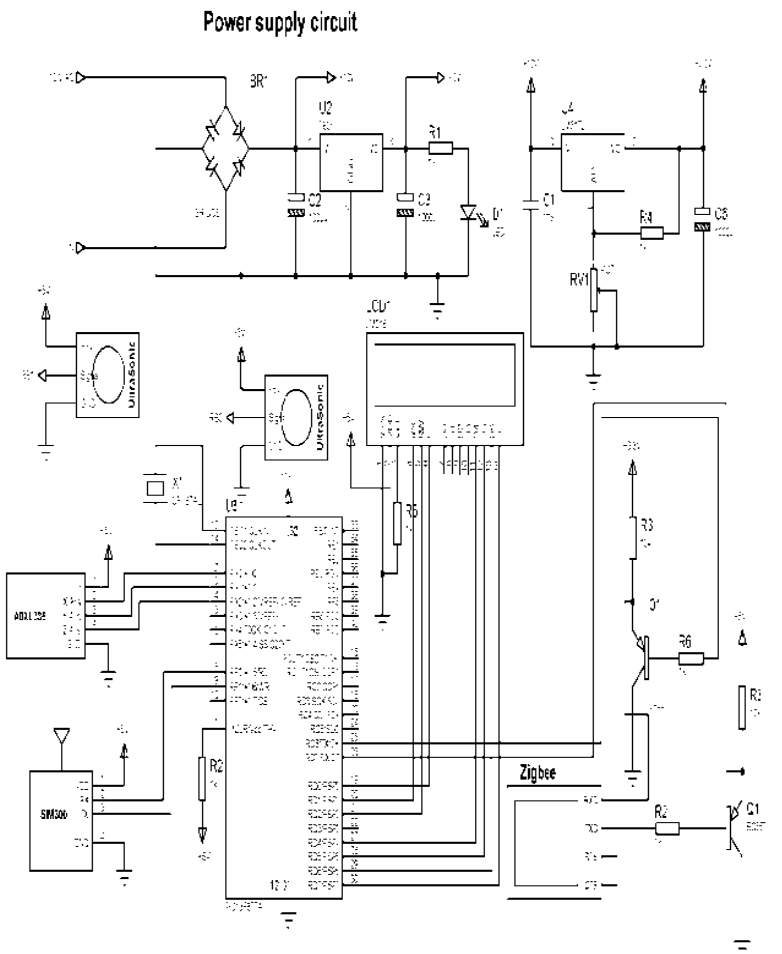

Fig. 4 Circuit diagram of ship detection

Operating on a single $1.8 \mathrm{~V}$ to $3.6 \mathrm{~V}$ supply, the ADXL335 consumes $350 \mu \mathrm{A}$. Available in a 16-lead LFCSP package; it is specified from $-40^{\circ} \mathrm{C}$ to $+85^{\circ} \mathrm{C}$. The pins are connected to RA0, RA1, and RA2 of the controller. The microcontroller PIC 16F877A is programmed where the analog signals are converted to digital form. The supply voltage of micro controller is $5 \mathrm{~V}$ and its oscillator frequency is $40 \mathrm{MHz}$ these digital signals are transmitted through Zigbee, whose operating voltage is $3.3 \mathrm{~V}$

\section{RESULTS AND DISCUSSION}

If the ship arrives, when the sensor senses the value above the set value alert levels is indicated.

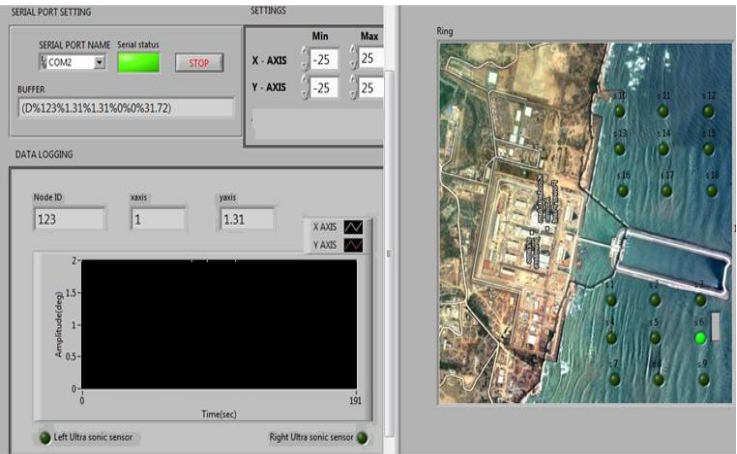

Fig.5 Snapshot of LabVIEW when ship not Arrives

Fig.5 shows the snapshot of LabVIEW when the ship is not arriving. During the normal conditions the sensors does not detect any of the values that are greater than the set limit. The graph shows the uniform oscillations of the normal sea waves and it visually monitors that there is no change in the values.
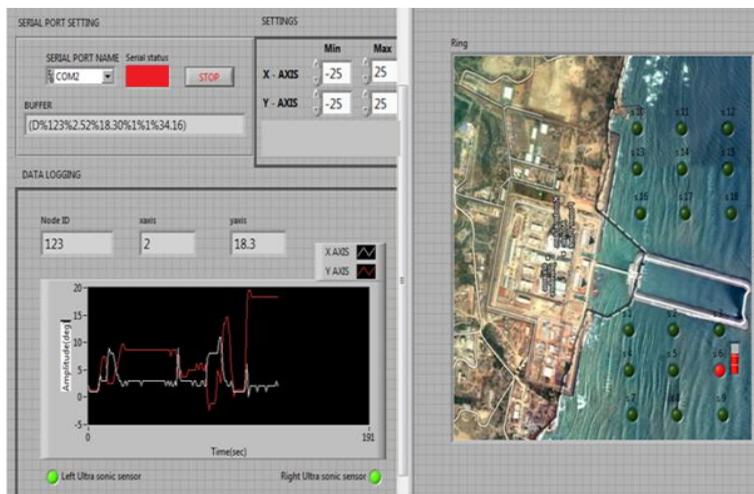

Fig.6 Snapshot of LabVIEW when ship Arrives

Fig.6 shows if the ship arrives, when the sensor senses the value above the set value alert levels is indicated. There are two alert levels. The first level indication is accelerometer sensor, with this input we cannot able to predict the intruder. So with the ultrasonic sensor as second level indication we able to decide that there is an intruder present in the border. The graphs visually shows the variations of ultrasonic and accelerometer sensors. According to the sensor values the alert level gets automatically active and buzzers are used to indicate the presences of intruder in the sea.

\section{CONCLUSION}

This paper gives the innovative solution for the people who cross over the border and saves more lives. The input is given to the microcontroller and it is transmitted through Zigbee. The transmitted signal from the Zigbee is received by the receiver node of the Zigbee and the output is displayed as graphical representation in LabVIEW. An alert message is send through mobile to the 
INTERNATIONAL JOURNAL OF INNOVATIVE RESEARCH IN ELECTRICAL, ELECTRONICS, INSTRUMENTATION AND CONTROL ENGINEERING Vol. 2, Issue 10, October 2014

coastal areas using GSM and database can be maintained in the personal computer. The overall accuracy of the system is good compared with the RADAR method.. This can be further extended by measuring the weight of the ship based on the height of sea waves.

\section{REFERENCES}

[1] A.T.Chwang \& Y.Chen (2003), 'Field Measurement of Ship Waves in Victoria Harbor', J.Eng.Mechanics, Vol.129, PP.1138-1148.

[2] M.Duarte\&Y.Hen Hu (2004), 'Vehicle Classification in Distributed SensorNetworks',

[3] S.Kumar,T.Lai\&A.Arora (2005), 'Barrier Coverage with Wireless Sensors,' PP.284-298.

[4] H.Zhu \& M.Ni (2008), 'HERO: Online Real Time Vehicle Tracking in Shanghai’, PP.942-950.

[5] E.Carapezza, J.Butman, I.Bab \& A. Bucklin (2008), 'Sustainable Coastal Sensor Networks: Technologies And Challenges', Vol.6963. 